\title{
An Extension of Cross Redundancy of Interval Scale Outputs and Inputs in DEA
}

\author{
Farhad Hosseinzadeh-Lotfi, ${ }^{1}$ Gholam-Reza Jahanshahloo, ${ }^{2}$ and Mansour Mohammadpour ${ }^{1}$ \\ ${ }^{1}$ Department of Mathematics, Science and Research Branch, Islamic Azad University, Hesarak, Poonak, Tehran, Iran \\ ${ }^{2}$ Faculty of Mathematical Science and Computer Engineering, University for Teacher Education, 599 Taleghani Avenue, \\ Tehran 15618, Iran
}

Correspondence should be addressed to Mansour Mohammadpour; mansour_mohammadpour@yahoo.com

Received 19 November 2012; Accepted 22 May 2013

Academic Editor: Hadi Nasseri

Copyright (C) 2013 Farhad Hosseinzadeh-Lotfi et al. This is an open access article distributed under the Creative Commons Attribution License, which permits unrestricted use, distribution, and reproduction in any medium, provided the original work is properly cited.

\begin{abstract}
It is well known that data envelopment analysis (DEA) models are sensitive to selection of input and output variables. As the number of variables increases, the ability to discriminate between the decision making units (DMUs) decreases. Thus, to preserve the discriminatory power of a DEA model, the number of inputs and outputs should be kept at a reasonable level. There are many cases in which an interval scale output in the sample is derived from the subtraction of nonnegative linear combination of ratio scale outputs and nonnegative linear combination of ratio scale inputs. There are also cases in which an interval scale input is derived from the subtraction of nonnegative linear combination of ratio scale inputs and nonnegative linear combination of ratio scale outputs. Lee and Choi (2010) called such interval scale output and input a cross redundancy. They proved that the addition or deletion of a cross-redundant output variable does not affect the efficiency estimates yielded by the CCR or BCC models. In this paper, we present an extension of cross redundancy of interval scale outputs and inputs in DEA models. We prove that the addition or deletion of a cross-redundant output and input variable does not affect the efficiency estimates yielded by the CCR or BCC models.
\end{abstract}

\section{Introduction}

In many DEA applications, such as income, an interval scale output in the sample is derived from the subtraction of nonnegative linear combination of ratio scale outputs and nonnegative linear combination of ratio scale inputs. There are also many cases, like cost, in which an interval scale input is derived from the subtraction of nonnegative linear combination of ratio scale inputs and nonnegative linear combination of ratio scale outputs, although the effect of such dependencies on DEA is not clear. Lee and Choi [1] called such interval scale output and input a cross redundancy. They proved that the addition or deletion of a cross-redundant output variable does not affect the efficiency estimates yielded by the CCR or BCC models. Francisco J. López [2] generalized the contributions of Lee and Choi by introducing specific definitions and conducting some additional analysis on the impact of the presence of other types of linear dependencies among the inputs and outputs of a DEA model. In this paper, we deal with cross-redundant output and input variables simultaneously in DEA models. We prove that the addition or deletion of a cross-redundant output and input variable does not affect the efficiency estimates yielded by the CCR or BCC models. The paper is organized as follows. In Section 2, we introduce preliminaries of DEA. In Section 3, we present our main results. In Section 4, we will illustrate that the addition or deletion of cross-redundant output variable and input variable does not affect the efficiency estimates yielded by the CCR or BCC models. Conclusions are summarized in Section 5 .

\section{Preliminaries}

Suppose that we have $n \geq 2$ peer observed DMUs, $\left\{\mathrm{DMU}_{j}\right.$ : $j=1,2, \ldots, n\}$ which produce multiple outputs $y_{r j},(r=$ 
$1, \ldots, s)$, by utilizing multiple inputs $x_{i j},(i=1, \ldots, m)$. The input and output vectors of $\mathrm{DMU}_{j}$ are denoted by $\mathbf{x}_{j}$ and $\mathbf{y}_{j}$, respectively, and we assume that $\mathbf{x}_{j}$ and $\mathbf{y}_{j}$ are semipositive, that is, $\mathbf{x}_{j} \geq 0, \mathbf{x}_{j} \neq 0$ and $\mathbf{y}_{j} \geq 0, y_{j} \neq 0$ for $i=1, \ldots, n$. We use $\left(\mathbf{x}_{j}, \mathbf{y}_{j}\right)$ to descript DMU $\mathrm{DM}_{j}$ and specially use $\left(\mathbf{x}_{o}, \mathbf{y}_{o}\right)$ (o element of $\left.\{1,2, \ldots, n\}\right)$ as the DMU under evaluation. Throughout this paper, vectors will be denoted by bold letters.

The input-oriented CCR [3] multiplier model evaluates the efficiency of each $\mathrm{DMU}_{o}$ by solving the following linear program:

$$
\begin{aligned}
& \theta^{*}=\max \quad \mathbf{u}^{t} \mathbf{y}_{o}, \\
& \mathbf{v}^{t} \mathbf{x}_{o}=1 \\
& \text { s.t. } \quad \mathbf{u}^{t} \mathbf{y}_{j} \leq \mathbf{v}^{t} \mathbf{x}_{j}, \quad j=1, \ldots, n, \\
& \mathbf{u} \geq o, \mathbf{v} \geq o .
\end{aligned}
$$

Because $\mathbf{x}_{j}$ and $\mathbf{y}_{j}$ are semipositive for $j=1, \ldots, n, \theta^{*}>0$. Also since $\mathbf{u}^{t} \mathbf{y}_{o} \leq \mathbf{v}^{t} \mathbf{x}_{o}$ and $\mathbf{v}^{t} \mathbf{x}_{o}=1$, we have $\theta^{*} \leq 1$. Thus $0<\theta^{*} \leq 1 . \theta^{*}$ represents the input-oriented CCR-efficiency value of $\mathrm{DMU}_{0}$.

The output-oriented CCR multiplier model evaluates the efficiency of each $\mathrm{DMU}_{o}$ by solving the following linear program:

$$
\begin{gathered}
\varphi^{*}=\min \quad \mathbf{v}^{t} \mathbf{x}_{o}, \\
\mathbf{u}^{t} \mathbf{y}_{o}=1 \\
\text { s.t. } \quad \mathbf{v}^{t} \mathbf{x}_{j} \geq \mathbf{u}^{t} \mathbf{y}_{j}, \quad j=1, \ldots, n, \\
\mathbf{u} \geq 0, \mathbf{v} \geq 0 .
\end{gathered}
$$

Since $\mathbf{u}^{t} \mathbf{y}_{o} \leq \mathbf{v}^{t} \mathbf{x}_{o}$ and $\mathbf{u}^{t} \mathbf{y}_{o}=1$, we have $\varphi^{*} \geq 1$. $1 / \varphi^{*}$ represents the output-oriented CCR-efficiency value of $\mathrm{DMU}_{o}$. Also $\theta^{*}=1 / \varphi^{*}[4]$.

The input-oriented BCC [4] multiplier model evaluates the efficiency of each $\mathrm{DMU}_{o}$ by solving the following linear program:

$$
\begin{aligned}
& z^{*}=\max \mathbf{u}^{t} \mathbf{y}_{o}+u_{o}, \\
& \mathbf{v}^{t} \mathbf{x}_{o}=1 \\
& \text { s.t. } \quad \mathbf{u}^{t} \mathbf{y}_{j}+u_{o} \leq \mathbf{v}^{t} \mathbf{x}_{j}, \quad j=1, \ldots, n, \\
& \mathbf{u} \geq \mathbf{0}, \mathbf{v} \geq \mathbf{0}, u_{o} \text { is free. }
\end{aligned}
$$

Let $\left(\mathbf{u}^{*}, \mathbf{v}^{*}\right)$ be an optimal feasible solution for model (1); then $\left(\mathbf{u}^{*}, \mathbf{v}^{*}, u_{o}^{*}\right)$, where $u_{o}^{*}=0$, will be a feasible solution of model (3). Thus $z^{*} \geq \theta^{*}$; therefore, $0<z^{*} \leq 1$. $z^{*}$ represents the input-oriented BCC-efficiency value of $\mathrm{DMU}_{o}$.
Finally, the output-oriented BCC multiplier model evaluates the efficiency of each $\mathrm{DMU}_{o}$ by solving the following linear program:

$$
\begin{aligned}
& t^{*}=\min \mathbf{v}^{t} \mathbf{x}_{o}-v_{o}, \\
& \mathbf{u}^{t} \mathbf{y}_{o}=1 \\
& \text { s.t. } \quad \mathbf{v}^{t} \mathbf{x}_{j}-v_{o} \geq \mathbf{u}^{t} \mathbf{y}_{j}, \quad j=1, \ldots, n, \\
& \mathbf{u} \geq \mathbf{0}, \mathbf{v} \geq \mathbf{0}, \quad v_{o} \text { is free. }
\end{aligned}
$$

It can be easily confirmed that $t^{*} \geq 1.1 / t^{*}$ represents the output-oriented BCC-efficiency value of $\mathrm{DMU}_{o}$.

\section{Main Results}

In this section, we prove that the addition or deletion of a cross-redundant output variable and/or input variable does not affect the efficiency estimates yielded by the BCC multiplier model in input- and output-oriented versions. Similarly, it can be proved that the addition or deletion of cross-redundant variable does not affect efficiency estimates yielded by the CCR multiplier model in input- and outputoriented versions.

Theorem 1. Let each DMU have $m+1$ inputs and $s+1$ outputs, that is, $\mathbf{x}_{j}=\left(x_{1 j}, \ldots, x_{(m+1) j}\right)$ and $\mathbf{y}_{j}=\left(y_{1 j}, \ldots, y_{(s+1) j}\right)$ for $j=1,2, \ldots, n$. Let

$$
\begin{array}{r}
x_{(m+1) j}=\sum_{i=1}^{m} \beta_{i} x_{i j}-\sum_{r=1}^{s} \alpha_{r} y_{r j} ; \quad j=1, \ldots, n, \\
y_{(s+1) j}=\sum_{r=1}^{s} a_{r} y_{r j}-\sum_{i=1}^{m} b_{i} x_{i j} ; \quad j=1, \ldots, n,
\end{array}
$$

where $\beta_{i} \geq 0, b_{i} \geq 0, i=1, \ldots, m ; \alpha_{r} \geq 0, a_{r} \geq 0, r=$ $1, \ldots, s$.

Then the optimal objective function value of the following model:

$$
\begin{aligned}
\rho^{*}=\max & \sum_{r=1}^{s+1} p_{r} y_{r o}+p_{o} \\
& \sum_{i=1}^{m+1} q_{i} x_{i o}=1, \\
\text { s.t } \quad & \sum_{r=1}^{s+1} p_{r} y_{r o}-\sum_{i=1}^{m+1} q_{i} x_{i o}-p_{o} \leq 0, \quad j=1, \ldots, n,
\end{aligned}
$$

$$
p_{r} \geq 0, \quad q_{i} \geq 0, \quad r=1, \ldots, s+1, i=1, \ldots, m+1
$$

is equal to the optimal objective function value of the following model (3). 
Proof. Let $\left(p_{1}^{*}, \ldots, p_{s+1}^{*}, q_{1}^{*}, \ldots, q_{m+1}^{*}, p_{o}^{*}\right)$ be an optimal solution for model (7); then we have

$$
\begin{gathered}
\rho^{*}=\sum_{r=1}^{s+1} p_{r}^{*} y_{r o}-p_{o}^{*}, \\
\sum_{i=1}^{m+1} q_{i}^{*} x_{i o}=1, \\
\sum_{r=1}^{s+1} p_{r}^{*} y_{r o}-\sum_{i=1}^{m+1} q_{i}^{*} x_{i o}-p_{o}^{*} \leq 0 .
\end{gathered}
$$

By (6) and (9), it follows that

$$
\rho^{*}=\sum_{r=1}^{s}\left(p_{r}^{*}+p_{s+1}^{*}\right) y_{r o}-\sum_{i=1}^{m} p_{s+1}^{*} b_{i} x_{i o}+p_{o}^{*} .
$$

Also, by (5) and (9) it concludes that

$$
\sum_{i=1}^{m}\left(q_{i}^{*}+q_{m+1}^{*} \beta_{i}\right) x_{i o}-\sum_{r=1}^{s} q_{m+1}^{*} \alpha_{r} y_{r o}=1
$$

Now, let

$$
\begin{gathered}
\bar{v}_{i}=\frac{\left(q_{i}^{*}+q_{m+1}^{*} \beta_{i}\right) \rho^{*}}{A B}+\frac{p_{s+1}^{*} b_{i}}{A}, \quad \text { for } i=1, \ldots, m, \\
\bar{u}_{r}=\frac{\left(p_{r}^{*}+p_{s+1}^{*} a_{r}\right) \rho^{*}}{A B}+\frac{\left(q_{m+1}^{*} \alpha_{r}\right) \rho^{*}}{B}, \text { for } i=1, \ldots, m, \\
\bar{u}_{o}=\frac{p_{o}^{*} \rho^{*}}{A B},
\end{gathered}
$$

where

$$
\begin{gathered}
A=\sum_{r=1}^{s}\left(p_{r}^{*}+p_{s+1}^{*} a_{r}\right) y_{r o}+p_{o}^{*} \\
B=1+\sum_{r=1}^{s} q_{m+1}^{*} \alpha_{r} y_{r o} .
\end{gathered}
$$

Then, by (7), we have

$$
\bar{v}_{i} \geq 0, \quad i=1, \ldots, m, \quad \bar{u}_{r} \geq 0, \quad r=1, \ldots, s .
$$

Also, by (12) and (13), we obtain

$$
\begin{aligned}
& \sum_{i=1}^{m} \bar{v}_{i} x_{i o}=\frac{\rho^{*}}{A B} \sum_{i=1}^{m}\left(q_{i}^{*}+q_{m+1}^{*} \beta_{i}\right) x_{i o} \\
& \quad+\frac{1}{A} \sum_{i=1}^{m}\left(p_{s+1}^{*} b_{i}\right) x_{i o}=\frac{\rho^{*}(B)}{A B}+\frac{1}{A}\left(A-\rho^{*}\right)=1 .
\end{aligned}
$$

In addition,

$$
\begin{aligned}
\theta^{*} \geq & \sum_{r=1}^{s} \bar{u}_{r} y_{r o}-\bar{u}_{o}=\frac{\rho^{*}}{A B} \sum_{r=1}^{s}\left(p_{r}^{*}+p_{s+1}^{*} a_{r}\right) y_{r o} \\
& +\frac{z_{2}^{*}}{B} \sum_{r=1}^{s}\left(q_{m+1}^{*} \alpha_{r}\right)-\frac{p_{o}^{*} \rho^{*}}{A B} \\
= & \frac{\rho^{*}}{A B}\left(\sum_{r=1}^{s}\left(p_{r}^{*}+p_{s+1}^{*} a_{r}\right) y_{r o}-p_{o}^{*}\right)+\frac{\rho^{*}(B-1)}{B} \\
= & \frac{z_{2}^{*}}{A B}(A)+\frac{\rho^{*}(B-1)}{B}=\rho^{*} .
\end{aligned}
$$

Also

$$
\begin{aligned}
\sum_{i=1}^{m} \bar{v}_{i} x_{i j} & -\sum_{r=1}^{s} \bar{u}_{r} y_{r j}+\bar{u}_{o} \\
= & \frac{1}{A B} \sum_{i=1}^{m} \rho^{*}\left(q_{i}^{*}+q_{m+1}^{*} \beta_{i}\right) x_{i j}+\frac{1}{A} \sum_{i=1}^{m} p_{s+1}^{*} b_{i} x_{i j} \\
& -\frac{1}{A B} \sum_{r=1}^{s} \rho^{*}\left(p_{r}^{*}+p_{s+1}^{*} a_{r}\right) y_{r j} \\
& -\frac{1}{B} \sum_{r=1}^{s} \rho^{*}\left(q_{m+1}^{*} \alpha_{r}\right) y_{r j}+\frac{1}{A B} p_{o}^{*} \rho^{*} \\
= & \frac{1}{A B}\left[\rho^{*}\left(\sum_{i=1}^{m} q_{i}^{*} x_{i j}-\sum_{r=1}^{s} p_{r}^{*} y_{r j}+p_{o}^{*}\right)\right.
\end{aligned}
$$

$$
\begin{aligned}
& +\rho^{*}\left(\sum_{i=1}^{m} q_{m+1}^{*} \beta_{i} x_{i j}-\sum_{r=1}^{s} p_{s+1}^{*} a_{r} y_{r j}\right) \\
& \left.+B \sum_{i=1}^{m} p_{s+1}^{*} b_{i} x_{i j}-A \rho^{*} \sum_{r=1}^{s}\left(q_{m+1}^{*} \alpha_{r}\right) y_{r j}\right] .
\end{aligned}
$$

So that by (10) we have

$$
\begin{aligned}
& \sum_{i=1}^{m} \bar{v}_{i} x_{i j}-\sum_{r=1}^{s} \bar{u}_{r} y_{r j}+\bar{u}_{o} \\
& \geq \frac{1}{A B}\left[\rho ^ { * } \left(\sum_{r=1}^{s}\left(p_{s+1}^{*} a_{r}+q_{m+1}^{*} \alpha_{r}\right) y_{r j}\right.\right. \\
&\left.\quad-\sum_{i=1}^{m}\left(q_{m+1}^{*} \beta_{i}+p_{s+1}^{*} b_{i}\right) x_{i j}\right)
\end{aligned}
$$




$$
\begin{gathered}
+\rho^{*}\left(\sum_{i=1}^{m} q_{m+1}^{*} \beta_{i} x_{i j}-\sum_{r=1}^{s} p_{s+1}^{*} a_{r} y_{r j}\right) \\
\left.+B \sum_{i=1}^{m} p_{s+1}^{*} b_{i} x_{i j}-A \rho^{*} \sum_{r=1}^{s}\left(q_{m+1}^{*} \alpha_{r}\right) y_{r j}\right] \\
=\frac{1}{A B}\left[\rho^{*} \sum_{r=1}^{s} q_{m+1}^{*} \alpha_{r} y_{r j}-\rho^{*} \sum_{i=1}^{m} p_{s+1}^{*} b_{i} x_{i j}\right. \\
\left.+B \sum_{i=1}^{m} p_{s+1}^{*} b_{i} x_{i j}-A \rho^{*} \sum_{r=1}^{s}\left(q_{m+1}^{*} \alpha_{r}\right) y_{r j}\right] \\
=\frac{1}{A B}\left[\left(B-\rho^{*}\right) \sum_{i=1}^{m} p_{s+1}^{*} b_{i} x_{i j}+\rho^{*}(1-A) \sum_{r=1}^{s} q_{m+1}^{*} \alpha_{r} y_{r j}\right] .
\end{gathered}
$$

Therefore,

$$
\begin{aligned}
\sum_{i=1}^{m} \bar{v}_{i} x_{i j}- & \sum_{r=1}^{s} \bar{u}_{r} y_{r j}+\bar{u}_{o} \\
\geq \frac{1}{A B} & {\left[\left(B-\rho^{*}\right) \sum_{i=1}^{m} p_{s+1}^{*} b_{i} x_{i j}\right.} \\
& \left.+\rho^{*}(1-A) \sum_{r=1}^{s} q_{m+1}^{*} \alpha_{r} y_{r j}\right] \geq 0 .
\end{aligned}
$$

Consequently, $\left(\overline{\mathbf{u}}, \overline{\mathbf{v}}, \bar{u}_{o}\right)$, where $\overline{\mathbf{u}}=\left(\bar{u}_{1}, \ldots, \bar{u}_{s}\right)$ and $\overline{\mathbf{v}}=$ $\left(\bar{v}_{1}, \ldots, \bar{v}_{m}\right)$, is a feasible solution for model (1), which for.

$$
\begin{aligned}
\theta^{*} \geq & \sum_{r=1}^{s} \bar{u}_{r} y_{r o}-\bar{u}_{o}=\frac{\rho^{*}}{A B} \sum_{r=1}^{s}\left(p_{r}^{*}+p_{s+1}^{*} a_{r}\right) y_{r o} \\
& +\frac{z_{2}^{*}}{B} \sum_{r=1}^{s}\left(q_{m+1}^{*} \alpha_{r}\right)-\frac{p_{o}^{*} \rho^{*}}{A B} \\
= & \frac{\rho^{*}}{A B}\left(\sum_{r=1}^{s}\left(p_{r}^{*}+p_{s+1}^{*} a_{r}\right) y_{r o}-p_{o}^{*}\right)+\frac{\rho^{*}(B-1)}{B} \\
= & \frac{z_{2}^{*}}{A B}(A)+\frac{\rho^{*}(B-1)}{B}=\rho^{*} .
\end{aligned}
$$

Now, let $\left(\mathbf{u}^{*}, \mathbf{v}^{*}, u_{o}^{*}\right)$ be an optimal solution for model (1); then $\left(\overline{\mathbf{p}}, \overline{\mathbf{q}}, \bar{p}_{o}\right)$, where $\overline{\mathbf{p}}=\left(\bar{p}_{1}, \ldots, \bar{p}_{s+1}\right)$ and $\overline{\mathbf{q}}=$ $\left(\bar{q}_{1}, \ldots, \bar{q}_{m+1}\right)$, with $\bar{p}_{r}=\bar{u}_{r}, r=1, \ldots, s ; \bar{p}_{s+1}=0 ; \bar{q}_{i}=$ $\bar{v}_{i}, \quad i=1, \ldots, m ; \bar{q}_{m+1}=0 ; \bar{p}_{o}=u_{o}^{*}$, is a feasible solution for model (2), which for $\theta^{*}=\sum_{r=1}^{s} u_{r}^{*} y_{r o}-u_{o}^{*}=\sum_{r=1}^{s+1} \bar{p}_{r} y_{r o}-\bar{p}_{o} \leq$ $\rho^{*}$. Thus $\theta^{*}=\rho^{*}$.

Theorem 2. Let each DMU have $m+1$ inputs and $s+1$ outputs with conditions (5) and (6).
Then the optimal objective function value of the following model:

$$
\begin{aligned}
& w^{*}=\min \quad \sum_{r=1}^{s+1} q_{i} x_{i o}-q_{o}, \\
& \sum_{r=1}^{s+1} p_{r} y_{r o}=1 \\
& \text { s.t } \quad \sum_{i=1}^{m+1} q_{i} x_{i o}-\sum_{r=1}^{s+1} p_{r} y_{r o}-q_{o} \geq 0, \quad j=1, \ldots, n, \\
& p_{r} \geq 0, \quad q_{i} \geq 0, \quad r=1, \ldots, s+1, \quad i=1, \ldots, m+1
\end{aligned}
$$

is equal to the optimal objective function value of the following model (4).

Proof. Let $\left(p_{1}^{*}, \ldots, p_{s+1}^{*}, q_{1}^{*}, \ldots, q_{m+1}^{*}, q_{o}^{*}\right)$ be an optimal solution for model (22); then we have

$$
\begin{gathered}
\omega^{*}=\sum_{i=1}^{m+1} q_{i}^{*} x_{i o}-q_{o}^{*} \\
\sum_{r=1}^{s+1} p_{r}^{*} y_{r o}=1 \\
\sum_{i=1}^{m+1} q_{i}^{*} x_{i j}-\sum_{r=1}^{s} p_{r}^{*} y_{r j}-q_{o}^{*} \geq 0 .
\end{gathered}
$$

By (6) and (15), it follows that

$$
\omega^{*}=\sum_{i=1}^{m}\left(q_{i}^{*}+q_{m+1}^{*} \beta_{i}\right) x_{i o}-q_{o}^{*}-\sum_{r=1}^{s} q_{m+1}^{*} \alpha_{r} y_{r o} .
$$

Also, by (5) and (16) it concludes that

$$
\sum_{r=1}^{s}\left(p_{r}^{*}+p_{s+1}^{*} a_{r}\right) y_{r o}-\sum_{i=1}^{m} p_{s+1}^{*} b_{i} x_{i o}=1 .
$$

Now, let

$$
\begin{array}{ll}
\bar{v}_{i}=\frac{\left(q_{i}^{*}+q_{m+1}^{*} \beta_{i}\right) w^{*}}{A B}+\frac{w^{*} p_{s+1}^{*} b_{i}}{B}, & \text { for } i=1, \ldots, m, \\
\bar{u}_{r}=\frac{\left(p_{r}^{*}+p_{s+1}^{*} a_{r}\right) w^{*}}{A B}+\frac{\left(q_{m+1}^{*} \alpha_{r}\right)}{A}, & \text { for } r=1, \ldots, s, \\
\bar{v}_{o}=\frac{q_{o}^{*} w^{*}}{A B}, &
\end{array}
$$

where

$$
\begin{gathered}
A=\sum_{i=1}^{m}\left(q_{i}^{*}+q_{m+1}^{*} \beta_{i}\right) x_{i o}-q_{o}^{*}, \\
B=1+\sum_{r=1}^{s} q_{m+1}^{*} \alpha_{r} y_{r o} .
\end{gathered}
$$


Then, by (7), we have

$$
\begin{aligned}
& \bar{v}_{i} \geq 0, \quad i=1, \ldots, m \\
& \bar{u}_{r} \geq 0, \quad r=1, \ldots, s .
\end{aligned}
$$

Also, by (26) and (27), we obtain

$$
\begin{aligned}
\sum_{r=1}^{s} \bar{u}_{r} y_{r o}= & \frac{w^{*}}{A B} \sum_{i=1}^{m}\left(p_{r}^{*}+p_{s+1}^{*} \alpha_{r}\right) y_{r o} \\
& +\frac{w^{*}}{B} \sum_{i=1}^{m}\left(q_{m+1}^{*} \alpha_{r}\right) y_{r o}=\frac{w^{*}(B)}{A B}+\frac{\left(A-w^{*}\right)}{A}=1 .
\end{aligned}
$$

In addition

$$
\begin{aligned}
\sum_{i=1}^{m} \bar{v}_{i} x_{i j}- & \sum_{r=1}^{s} \bar{u}_{r} y_{r j}-\bar{v}_{o} \\
= & \frac{1}{A B} \sum_{i=1}^{m} w^{*}\left(q_{i}^{*}+q_{m+1}^{*} \beta_{i}\right) x_{i j} \\
+ & \frac{w^{*}}{B} \sum_{i=1}^{m} p_{s+1}^{*} b_{i} x_{i j} \\
& -\frac{w^{*}}{A B} \sum_{r=1}^{s}\left(p_{r}^{*}+p_{s+1}^{*} a_{r}\right) y_{r j} \\
& -\frac{1}{A} \sum_{r=1}^{s}\left(q_{m+1}^{*} \alpha_{r}\right) y_{r j}-\frac{1}{A B} q_{o}^{*} w^{*} \\
= & \frac{1}{A B}\left[w^{*}\left(\sum_{i=1}^{m} q_{i}^{*} x_{i j}-\sum_{r=1}^{s} p_{r}^{*} y_{r j}-q_{o}^{*}\right)\right. \\
& +w^{*}\left(\sum_{i=1}^{m} q_{m+1}^{*} \beta_{i} x_{i j}-\sum_{r=1}^{s} p_{s+1}^{*} a_{r} y_{r j}\right) \\
& \left.+w^{*} \sum_{i=1}^{m} p_{s+1}^{*} b_{i} x_{i j}-B \sum_{r=1}^{s}\left(q_{m+1}^{*} \alpha_{r}\right) y_{r j}\right]
\end{aligned}
$$

So that by (14), we have

$$
\begin{aligned}
\sum_{i=1}^{m} \bar{v}_{i} x_{i j}-\sum_{r=1}^{s} \bar{u}_{r} y_{r j}-\bar{v}_{o} \\
\geq \frac{1}{A B}\left[w ^ { * } \left(\sum_{r=1}^{s}\left(p_{s+1}^{*} a_{r}+q_{m+1}^{*} \alpha_{r}\right) y_{r j}\right.\right. \\
\left.-\sum_{i=1}^{m}\left(q_{m+1}^{*} \beta_{i}+p_{s+1}^{*} b_{i}\right) x_{i j}\right) \\
+w^{*}\left(\sum_{i=1}^{m} q_{m+1}^{*} \beta_{i} x_{i j}-\sum_{r=1}^{s} p_{s+1}^{*} a_{r} y_{r j}\right) \\
\left.+A w^{*} \sum_{i=1}^{m} p_{s+1}^{*} b_{i} x_{i j}-B \sum_{r=1}^{s}\left(q_{m+1}^{*} \alpha_{r}\right) y_{r j}\right]
\end{aligned}
$$

$$
\begin{gathered}
=\frac{1}{A B}\left[w^{*} \sum_{r=1}^{s} q_{m+1}^{*} \alpha_{r} y_{r j}-w^{*} \sum_{i=1}^{m} p_{s+1}^{*} b_{i} x_{i j}\right. \\
\left.+A w^{*} \sum_{i=1}^{m} p_{s+1}^{*} b_{i} x_{i j}-B \sum_{r=1}^{s}\left(q_{m+1}^{*} \alpha_{r}\right) y_{r j}\right] \\
=\frac{1}{A B}\left[w^{*}(A-1) \sum_{i=1}^{m} p_{s+1}^{*} b_{i} x_{i j}\right. \\
\left.+\left(w^{*}-B\right) \sum_{r=1}^{s} q_{m+1}^{*} \alpha_{r} y_{r j}\right] .
\end{gathered}
$$

Therefore,

$$
\begin{aligned}
\sum_{i=1}^{m} \bar{v}_{i} x_{i j}-\sum_{r=1}^{s} \bar{u}_{r} y_{r j}-\bar{v}_{o} \\
\geq \frac{1}{A B}\left[w^{*}(A-1) \sum_{i=1}^{m} p_{s+1}^{*} b_{i} x_{i j}\right. \\
\left.+\left(w^{*}-B\right) \sum_{r=1}^{s} q_{m+1}^{*} \alpha_{r} y_{r j}\right] \geq 0
\end{aligned}
$$

Consequently, $\left(\overline{\mathbf{u}}, \overline{\mathbf{v}}, \bar{v}_{o}\right)$, where $\overline{\mathbf{u}}=\left(\bar{u}_{1}, \ldots, \bar{u}_{s}\right)$ and $\overline{\mathbf{v}}=$ $\left(\bar{v}_{1}, \ldots, \bar{v}_{m}\right)$, is a feasible solution for model (4), which for

$$
\begin{aligned}
z^{*} \leq & \sum_{i=1}^{m} \bar{v}_{i} x_{i o}-\bar{v}_{o} \\
= & \frac{w^{*}}{A B} \sum_{i=1}^{m}\left(q_{i}^{*}+q_{m+1}^{*} \beta_{r}\right) x_{i o} \\
& +\frac{w^{*}}{B} \sum_{r=1}^{s}\left(p_{s+1}^{*} b_{i}\right) x_{i o}-\frac{q_{o}^{*} w^{*}}{A B} \\
= & \frac{w^{*}}{A B}(A)+\frac{w^{*}(B-1)}{B}=w^{*} .
\end{aligned}
$$

Now let $\left(\mathbf{u}^{*}, \mathbf{v}^{*}, v_{o}^{*}\right)$ be an optimal solution for model (4), and then $\left(\overline{\mathbf{p}}, \overline{\mathbf{q}}, \bar{p}_{o}\right)$, where $\overline{\mathbf{p}}=\left(\bar{p}_{1}, \ldots, \bar{p}_{s+1}\right)$ and $\overline{\mathbf{q}}=$ $\left(\bar{q}_{1}, \ldots, \bar{q}_{m+1}\right)$, with $\bar{p}_{r}=\bar{u}_{r}, r=1, \ldots, s ; \bar{p}_{s+1}=0 ; \bar{q}_{i}=$ $\bar{v}_{i}, i=1, \ldots, m ; \bar{q}_{m+1}=0 ; \bar{p}_{o}=v_{o}^{*}$, is a feasible solution for model (22), which for $\omega^{*} \geq \sum_{r=1}^{s+1} \bar{p}_{r} y_{r o}-\bar{p}_{o}=\sum_{r=1}^{s} u_{r}^{*} y_{r o}-$ $u_{o}^{*}=z^{*}$. Thus $z^{*}=w^{*}$.

Theorem 3. Let each DMU have $m+1$ inputs and $s+1$ outputs with conditions (5) and (6). 
Then, the optimal objective function value of the following model:

$$
\begin{aligned}
\tilde{\rho}=\max & \sum_{r=1}^{s+1} p_{r} y_{r o}, \\
& \sum_{i=1}^{m+1} q_{i} x_{i o}=1 \\
\text { s.t } \quad & \sum_{r=1}^{s+1} p_{r} y_{r j}-\sum_{i=1}^{m+1} q_{i} x_{i j} \leq 0, \quad j=1, \ldots, n \\
p_{r} \geq 0, \quad & q_{i} \geq 0, \quad r=1, \ldots, s+1, \quad i=1, \ldots, m+1
\end{aligned}
$$

is equal to the optimal objective function value of the following model (1).

Proof. This proof is similar to the proof of Theorem 1.

Theorem 4. Let each DMU have $m+1$ inputs and $s+1$ outputs with conditions (5) and (6).

Then, the optimal objective function value of the following model:

$$
\begin{array}{ll}
\widetilde{w}=\min & \sum_{r=1}^{s+1} q_{i} x_{i o}, \\
& \sum_{r=1}^{s+1} p_{r} y_{r o}=1 \\
\text { s.t } \quad \sum_{i=1}^{m+1} q_{i} x_{i j}-\sum_{r=1}^{s+1} p_{r} y_{r j} \geq 0, \quad j=1, \ldots, n, \\
p_{r} \geq 0, \quad q_{i} \geq 0, \quad r=1, \ldots, s+1, \quad i=1, \ldots, m+1
\end{array}
$$

is equal to the optimal objective function value of the following model (2).

Proof. This proof is similar to the proof of Theorem 2.

\section{Illustrative Example}

In this section, we use the data recorded in Table 1 to illustrate that the addition or deletion of a cross-redundant output variable and input variable does not affect the efficiency estimates yielded by the CCR or BCC models. These correspond to 20 DMUs, whose efficiency is assessed using four inputs and four outputs where

$$
\begin{gathered}
x_{4 j}=\left(x_{1 j}+x_{2 j}+2 x_{3 j}\right) \\
-\left(0.5 y_{1 j}+0.5 y_{2 j}+0.5 y_{3 j}\right) ; \\
j=1, \ldots, n, \\
y_{4 j}=\left(0.5 y_{1 j}+0.5 y_{2 j}+0.5 y_{3 j}\right)-0.5 x_{3 j} ; \\
j=1, \ldots, n .
\end{gathered}
$$

TABLE 1: Dataset.

\begin{tabular}{lcccccccc}
\hline & Inp 1 & Inp 2 & Inp 3 & Inp 4 & Out 1 & Out 1 & Out 3 & Out 4 \\
\hline Unit 1 & 7 & 1 & 4 & 12.75 & 1 & 2.5 & 3 & 1.25 \\
Unit 2 & 3 & 7 & 4 & 14.75 & 2.5 & 1 & 3 & 1.25 \\
Unit 3 & 6 & 6 & 3 & 14.25 & 2.5 & 2 & 3 & 2.25 \\
Unit 4 & 3 & 1 & 3 & 1.75 & 4 & 5.5 & 7 & 6.75 \\
Unit 5 & 6 & 0.5 & 3 & 5.25 & 5 & 3.5 & 6 & 5.75 \\
Unit 6 & 4 & 0.5 & 3 & 3.5 & 2 & 6 & 6 & 5.5 \\
Unit 7 & 1.5 & 2.5 & 3 & 1.5 & 6 & 4 & 7 & 7 \\
Unit 8 & 0.5 & 4 & 4 & 6.25 & 1.5 & 5 & 6 & 4.25 \\
Unit 9 & 2.75 & 1.75 & 4 & 4 & 8 & 3 & 6 & 6.5 \\
Unit 10 & 1 & 3 & 3 & 1 & 8 & 3 & 7 & 7.5 \\
Unit 11 & 2 & 2 & 3 & 1.25 & 5.5 & 5 & 7 & 7.25 \\
Unit 12 & 2.5 & 1.5 & 3 & 2 & 7 & 3 & 6 & 6.5 \\
Unit 13 & 4.5 & 1.5 & 6 & 13 & 4 & 2 & 4 & 2 \\
Unit 14 & 2 & 4 & 7 & 16.25 & 1.5 & 2 & 4 & 0.25 \\
Unit 15 & 4 & 3 & 6 & 12.25 & 6.5 & 3.5 & 3.5 & 3.75 \\
Unit 16 & 2 & 5 & 4 & 8.75 & 5 & 3.5 & 4 & 4.25 \\
Unit 17 & 1.5 & 6 & 4 & 8.5 & 4.5 & 4.5 & 5 & 5 \\
Unit 18 & 0.5 & 4 & 3 & 3 & 3.5 & 5.5 & 6 & 6 \\
Unit 19 & 3.5 & 0.75 & 3 & 2.5 & 7.5 & 2.5 & 6 & 6.5 \\
Unit 20 & 6 & 3.5 & 4 & 11 & 3.5 & 3.5 & 6 & 4.5 \\
\hline
\end{tabular}

TABLE 2: Example results.

\begin{tabular}{lcccc}
\hline & $\theta^{*}$ & $\tilde{\rho}$ & $z^{*}$ & $\rho^{*}$ \\
\hline Unit 1 & 0.3461538 & 0.3461538 & 0.7500000 & 0.7500000 \\
Unit 2 & 0.3214286 & 0.3214286 & 0.7500000 & 0.7500000 \\
Unit 3 & 0.4285714 & 0.4285714 & 1.0000000 & 1.0000000 \\
Unit 4 & 1.0000000 & 1.0000000 & 1.0000000 & 1.0000000 \\
Unit 5 & 1.0000000 & 1.0000000 & 1.0000000 & 1.0000000 \\
Unit 6 & 1.0000000 & 1.0000000 & 1.0000000 & 1.0000000 \\
Unit 7 & 1.0000000 & 1.0000000 & 1.0000000 & 1.0000000 \\
Unit 8 & 1.0000000 & 1.0000000 & 1.0000000 & 1.0000000 \\
Unit 9 & 0.9973190 & 0.9973190 & 1.0000000 & 1.0000000 \\
Unit 10 & 1.0000000 & 1.0000000 & 1.0000000 & 1.0000000 \\
Unit 11 & 1.0000000 & 1.0000000 & 1.0000000 & 1.0000000 \\
Unit 12 & 1.0000000 & 1.0000000 & 1.0000000 & 1.0000000 \\
Unit 13 & 0.4444444 & 0.4444444 & 0.6666667 & 0.6666667 \\
Unit 14 & 0.3809524 & 0.3809524 & 0.6666667 & 0.6666667 \\
Unit 15 & 0.5607702 & 0.5607702 & 0.5714286 & 0.5594240 \\
Unit 16 & 0.6052279 & 0.6052279 & 0.7500000 & 0.7500000 \\
Unit 17 & 0.6847156 & 0.6847156 & 0.7500000 & 0.7500000 \\
Unit 18 & 1.0000000 & 1.0000000 & 1.0000000 & 1.0000000 \\
Unit 19 & 1.0000000 & 1.0000000 & 1.0000000 & 1.0000000 \\
Unit 20 & 0.6428571 & 0.6428571 & 0.7500000 & 0.7500000 \\
\hline & & & &
\end{tabular}

In other words, the forth input and the forth output are crossredundant variables. In Table $2, \theta^{*}, \widetilde{z}, \rho^{*}$, and $\widetilde{\rho}$, respectively, record the efficiency measure provided by model (1), model (3), model (7), and model (36). It is evident from Table 2 that the addition or deletion of cross-redundant output variable 
and/or input variable does not affect the efficiency estimates yielded by the input-oriented CCR or BCC multiplier models.

\section{Conclusions}

In this paper, we have studied the effect of the cross redundancy between interval scale input and output variables on the efficiency estimates yielded by the CCR multiplier model in input- and output-oriented versions and the BCC multiplier model in input- and output-oriented versions. We proved that the addition or deletion of a cross-redundant output variable and input variable does not affect the efficiency estimates yielded by the input-oriented BCC multiplier model and the output-oriented BCC multiplier model. Similarly, it can be proved that the addition or deletion of cross-redundant variable does not affect efficiency estimates yielded by the CCR multiplier model in input- and outputoriented versions.

\section{References}

[1] K. Lee and K. Choi, "Cross redundancy and sensitivity in DEA models," Journal of Productivity Analysis, vol. 34, no. 2, pp. 151$165,2010$.

[2] F. J. López, "Generalizing cross redundancy in data envelopment analysis," European Journal of Operational Research, vol. 214, no. 3, pp. 716-721, 2011.

[3] A. Charnes, W. W. Cooper, and E. Rhodes, "Measuring the efficiency of decision making units," European Journal of Operational Research, vol. 2, no. 6, pp. 429-444, 1978.

[4] R. D. Banker, A. Charnes, and W. W. Cooper, "Some models for estimating technical and scale inefficiencies in data envelopment analysis," Management Science, vol. 30, no. 9, pp. 10781092, 1984. 


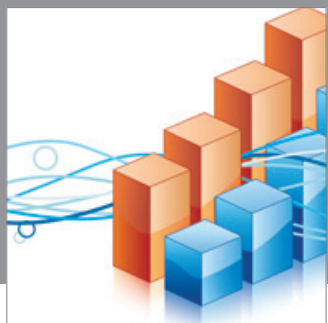

Advances in

Operations Research

mansans

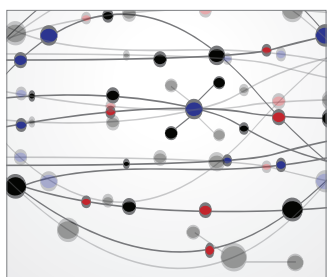

The Scientific World Journal
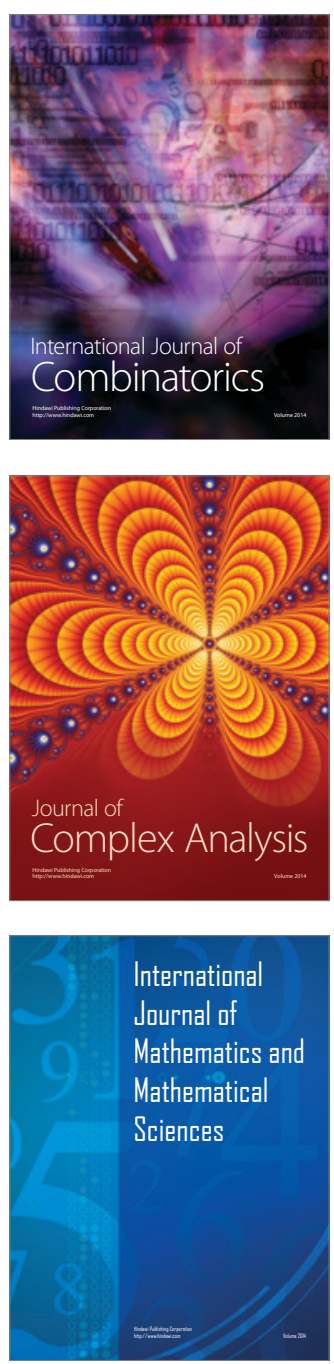
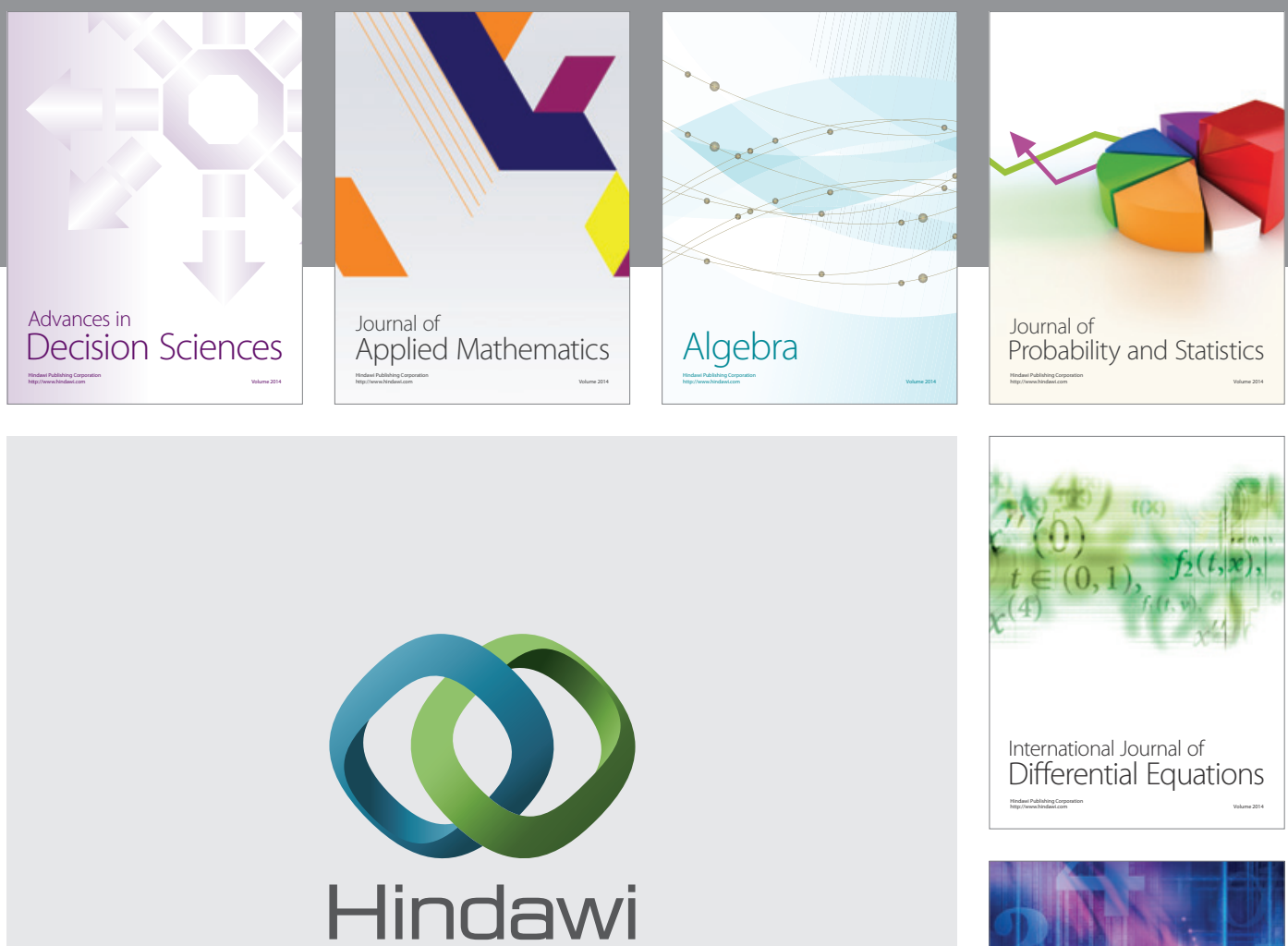

Submit your manuscripts at http://www.hindawi.com
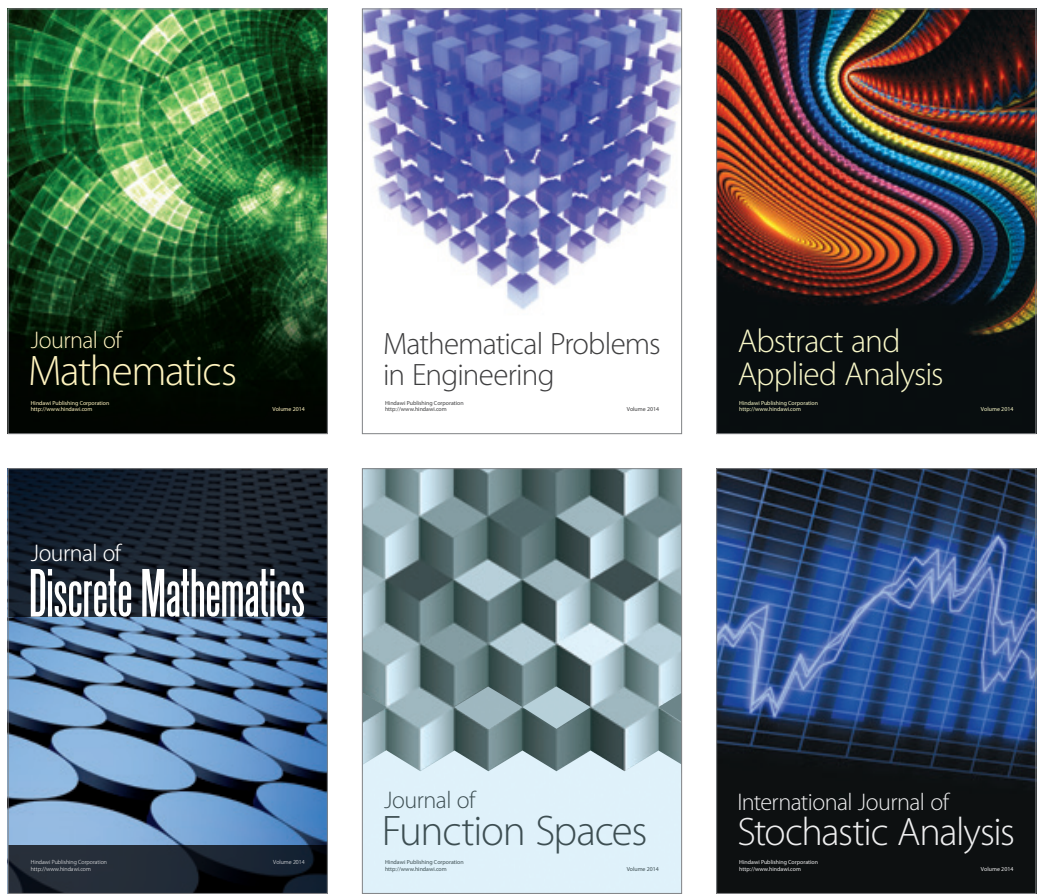

Journal of

Function Spaces

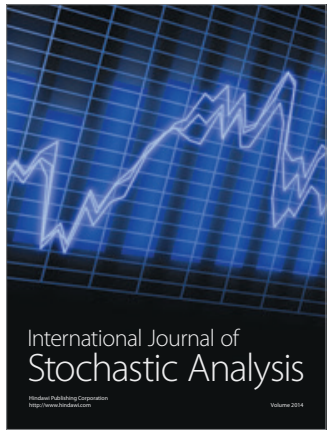

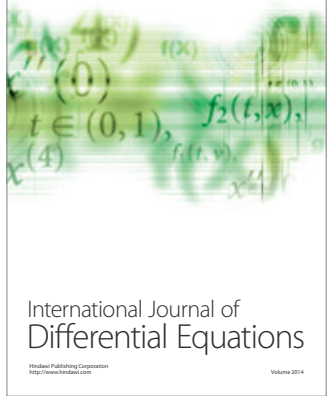
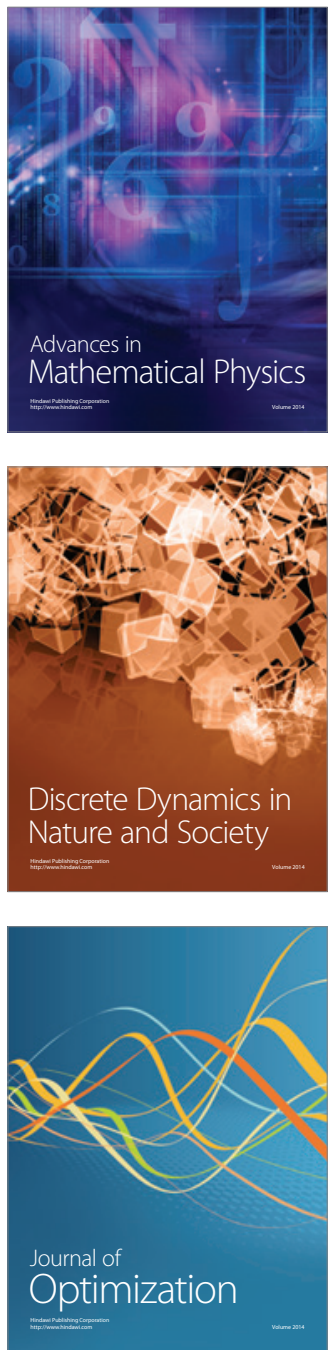